\title{
Etablierung eines Brustzentrums in einer Flächenregion: Brustzentrum Altmark
}

\author{
Stephan Henschen \\ Brustzentrum, Johanniter-Krankenhaus Genthin-Stendal, Germany
}

Zentrenbildung liegt im Trend - aber wie können Lösungen für dünn besiedelte Regionen aussehen? Aufgrund der europäischen Entwicklung und der deutschen Bemühungen zu Brustzentren hin haben wir die Notwendigkeit erkannt, auch in der Altmark ein Brustzentrum zu etablieren. Die Versorgung der Mammakarzinom-Patientinnen, aber auch der Frauen und Männer mit gutartigen Veränderungen in der Brust, stellt in der Flächenregion ein großes Problem dar. Durch große Entfernungen und eine geringe Bevölkerungsdichte in der Region ist die wohnortnahe Versorgung nicht umzusetzen. Die technischen Anforderungen an ein Brustzentrum sind hoch und machen ökonomisch schwer zu handhabende Investitionen notwendig. Die Qualifizierung der eigenen Mitarbeiter (Onkologiefachschwester, spezielle Weiterbildungen für Mammographie, Psychoonkologie, Palliativmedizin, Schmerztherapie und vieles mehr), die Optimierung der räumlichen Ausstattung (Atmosphäre im Chemotherapiezimmer, ansprechende Ambulanz, freundliche Räume) und weitere Gesichtspunkte sind Vorraussetzungen, um die Genesung der Patienten zu beschleunigen. Es ist nicht möglich, alle Leistungen an jedem Standort bereitzustellen. Hier ist dringend eine Bündelung der Ressourcen aber auch der fachlichen Qualifikation notwendig.

Als ich im Jahr 2000 aus Hamburg nach Stendal kam, war ich erschrocken über die vorhandenen Strukturen. Die Rate an brusterhaltenden Operationen war sehr gering (Abb. 1), und die Behandlung der Patienten war nicht organisiert und strukturiert. Üblicherweise führten Chirurgen die Mammaoperationen durch; überwiegend erfolgte die Brustamputation und die Ausräumung der Achelhöhlenlymphknoten. Die systemische Therapie lag im Ermessen des Einzelnen, wobei die Leitlinien häufig nicht umgesetzt wurden.

Da in der Universitätsklinik Hamburg Eppendorf bereits vor mehreren Jahrzehnten ein Brustzentrum etabliert war, in dem zweimal wöchentlich alle Brustkrebspatientinnen zeitnah und interdisziplinär (Strahlentherapeut, Diagnostiker, Operateur, Pathologe und Onkologe) besprochen wurden und ich auch entsprechend dort ausgebildet wurde, war für mich die vorgefundene Situation dringend veränderungsbedürftig.

Der Startschuss für das Brustzentrum Altmark fiel im Jahr 2002 in der Kurklinik Kalbe, die zwischen Stendal, Salzwedel und Gardelegen liegt. Ziel war es, alle drei in der Region aktiven Frauenkliniken zu vereinen, um gemeinsam die für die Zertifizierung notwendigen Strukturen zu schaffen. Auf Grund der geringen Fallzahlen in den drei Standorten mussten wir jedoch auf eine Zweistandortvariante ausweichen. Da die Kliniken in Gardelegen und Salzwedel unter einer gemeinsamen Trägerschaft stehen, war diese Lösung in vieler Hinsicht einfacher umzusetzen. Glücklicherweise waren die Ökonomen an beiden Standorten von dem Konzept überzeugt, so dass auch die notwendigen Investitionen getätigt werden konnten. Dadurch verfügen wir heute über die Möglichkeit der Stereotaxie sowohl bei der Mammographie als auch beim MRT. Hierdurch konnte auch die Rate der offenen Biopsien nochmals deutlich gesenkt werden. Das Besondere ist, dass beide Standorte sich nicht als Konkurrenz verstehen, sondern die gewonnen Synergieeffekte zu nutzen wissen. So werden spezielle Leistungen jeweils nur an einem Standort vorgehalten und die Patienten entsprechend weitergeleitet. Trotz der großen Entfernung von $70 \mathrm{~km}$ zwischen Stendal und Salzwedel gelang es uns, alle Fachdisziplinen wöchentlich in den Brustboards zusammenzubringen, um die Therapien für jede Patientin festzulegen. Die Umsetzung der psychoonkologischen Betreuung ist durch die Kooperation mit der nahe gelegenen Klinik für Psychotherapie und Psychosomatik in Jerichow gelungen. Hier ließ sich schnell ein großes Engagement freisetzen. Der Aufbau einer sehr engen Kooperation auch durch gemeinsam besuchte Fortbildungsveranstaltungen hat das Verständnis für die Psychoonkologie wachsen lassen. Trotz einer Entfernung zwischen den Standorten von $20 \mathrm{~km}$

\begin{tabular}{ll}
\hline KARGER & @ 2007 S. Karger GmbH, Freiburg \\
Fax +497614520714 & Accessible online at: \\
$\begin{array}{l}\text { E-mail Information@Karger.de } \\
\text { www.karger.com }\end{array}$ & www.karger.com/brc
\end{tabular}

Dr. med. Stephan Henschen

Klinik für Frauenheilkunde und Geburtshilfe

Johanniter-Krankenhaus Genthin-Stendal gGmbH

Wendstraße 31, 39576 Stendal

Tel. +49 3931-660, Fax -217112

E-mail henschen@jksdl.de 
ist die schnelle Kontaktaufnahme bei aus Fragebögen ersichtlichem Bedarf jederzeit möglich. Die Struktur des Brustzentrums Altmark am Standort Stendal ist in Abbildung 2 dargestellt.

Seit 2000 nimmt die Klinik in Stendal an internationalen Studien teil, die Zahl der Studienpatienten konnte auf über 30\% gesteigert werden. Aufgrund der Anzahl der Patienten konnte eine Studynurse etabliert werden. Das Anwerben von interessanten Studien erfordert einen hohen zeitlichen Aufwand, auch hier ist ohne den persönlichen Einsatz der Einzelnen eine hohe Qualität nicht möglich.

Die Kraft die hinter der Verwirklichung des Projektes steht, hat in den Jahren des Bestehens nicht abgenommen. Der Zuspruch der Patientinnen ist sehr groß, was eine große Motivation ist. Der zeitliche Aufwand ist für alle Beteiligten sehr groß: Ohne persönliches Engagement und ohne geopferte Freizeit ist ein Brustzentrum in einer Flächenregion nicht umsetzbar, insbesondere da die Honorierung in zertifizierten und nicht zertifizierten Zentren gleich ist. Dass unser Brustzentrum sich dennoch etabliert hat, ist ein Beweis dafür, dass die Medizin noch nicht der Ökonomie zum Opfer gefallen ist. Im Zentrum unseres medizinischen Handelns sollte immer der gut aufgeklärte, psychisch stabile, nach den heute bestmöglichen Standards behandelte und hoffentlich am Ende gesunde Patient stehen. Wir hoffen nun, dass wir das bereits in der Theorie konzipierte Screeningprojekt auch praktisch erfolgreich durchführen werden, denn in Zukunft wird ein Brustzentrum ohne Screeningeinheit nicht umsetzbar sein. Dies gilt für beide Seiten.

Leider hat das Gesundheitssystem auch seine Tücken, an denen wir in den Flächenregionen nur schwer vorbeikommen. Die Etablierung einer eigenen Abteilung für Senologie ist bei den geringen Fallzahlen nicht möglich, die Behandlung der Patientinnen muss in das Gesamtkonzept der Klinik für Frauenheilkunde und Geburtshilfe integriert werden. Durch die Dezentralisierung der ambulanten Versorgung ist eine Einbindung der ambulant tätigen Ärzte schwer. Auch das Angebot regelmäßiger Weiterbildungen und die Möglichkeit an den Brustboards teilzunehmen, hat hier die persönlichen und über Jahrzehnte gewachsenen Strukturen nur schwer durchbrechen können. Obwohl nach anfänglichen Widerständen, z.B. gegen die brusterhaltende Therapie (z. Zt. 75\%), heute die Qualitätsentwicklung deutlich sichtbar ist, sind persönliche Ausbildung und fehlende Bindung an die Region noch immer ein

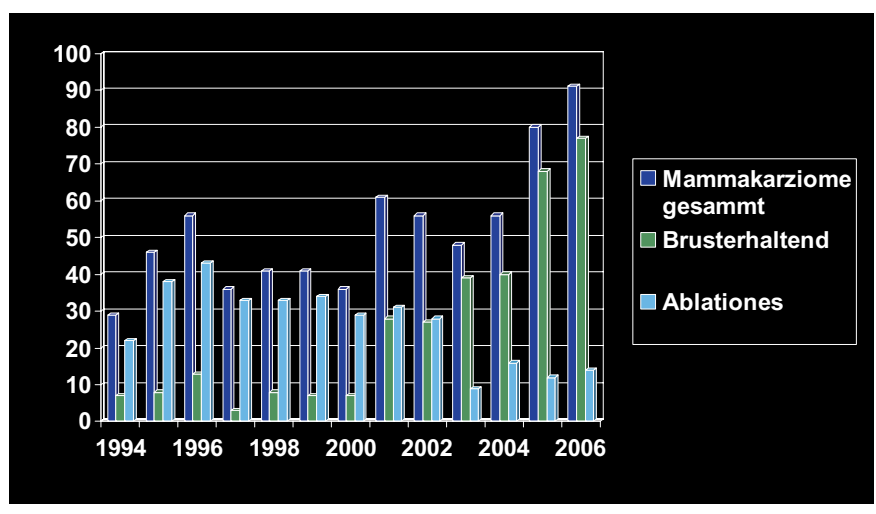

Abb. 1. Fallzahlenentwicklung im Brustzentrum Altmark, Standort Stendal.

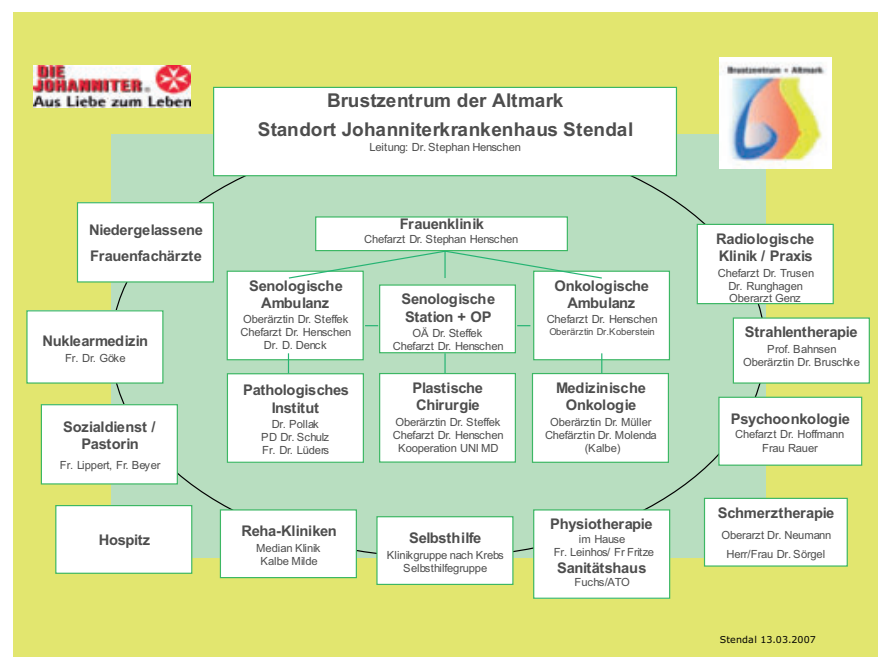

Abb. 2. Organisation des Brustzentrums Altmark am Standort Stendal.

Grund, Patienten in die Ballungszentren zu überweisen. Auch die Möglichkeit der Gründung von medizinischen Versorgungszentren wird diesen Effekt eventuell noch verstärken.

Die Bildung von Zentren führt sicher zu einer Verbesserung der Qualität, insbesondere in der sich schnell verändernden Behandlung des Mammakarzinoms. Die Entwicklung geht mehr zur individualisierten Behandlung. Um hier eine hervorragende Qualität anbieten zu können ist in der Flächenregion ein besonderes Engagement aller Beteiligten notwendig. Langfristig bleibt zu hoffen, dass der Fleiß Früchte trägt. 